\title{
Model of Social Networking Information System making use of User's Location and Interface for Mobile Computing
}

\author{
Apoorv Prasad \\ School of Information Technology and Engineering \\ VIT University \\ Vellore
}

\author{
Mahesh Kumar Haldar \\ School of Information Technology and Engineering \\ VIT University \\ Vellore
}

\begin{abstract}
Social Networking is one of the best platforms used by people all around the world. The main feature of it is to connect with friends (community, school, college, office etc.) and to make connections with the people from different parts of the world beyond geographical borders who have a common interest and want to share. But there are many issues with the current Social Networking Systems regarding privacy, data thefts, viruses, information misuse etc.

In our paper, we are focusing on coping up with the challenges of traditional social networking issues from a common user point of view. A common user wants to be introduced to other users, to get share mutual experiences and to connect with users of like orientations/interests.
\end{abstract}

\section{Keywords}

Verification, introduced, interface, professional, connections, Profiles, Portability.

\section{INTRODUCTION}

The social networking services are considered as an asset in today's world. It has become a common part of everyone's life. In 2014, there are about 1.82 billion social network users around the globe. It is expected that by the year 2017, the number of social network users will be 2.33 billion around the globe. The performance of social networking services are judged by the ease in getting in touch with the friends (classmates, schoolmates, colleagues), with the people who have a common interest and want to share. In our paper, we are not only focusing on improving the performance of social networking services but also resolving the social networking issues regarding privacy, data theft, viruses, information misuse etc.

In traditional model, User 1 can look for other User 2 by searching by their name, locations, Email-Id and can access their profile and get the information the User 1 want to extract from that. However, the features of privacy and security options have been made available in many social networking services, but not many users make use of that

Proposed model is that User 1 can look for User 2 based on their skills, events, communities and can get introduced by sending a request provided with a simple message. Also, one can easily make out the use of privacy shortcuts with the simple and easy interface provided for it.

\section{RELATED WORK}

Although progress has been made in this field for improving the social network services regarding: to look for the exact
User 2 by User 1 based on User 1's mutual friends, or some commonality in connections. The previous model didn't had the feature to get in touch with users or group of users based on the skills, events, communities governed by the location of the users.

\section{PROPOSED MODEL}

In the proceeding subsections we have discussed in detail the underlying components in the proposed model.

\subsection{User Registration}

As in every social networking platforms, the first and foremost thing is that the user makes his/her account by signing up and providing the mandatory details i.e. Name, Birthday (Month, Date and Year), Email Address, Location etc. Then, the activation link for the new account/user will be sent to the user's email id for the email address verification and other security verifications. After all the verification process the user can provide his/her additional details i.e. Profile Picture, Academic/Job Information, Skills etc. With the location mode "On", the user will be able to get friend's suggestions in and around his locality based on the information provided by him in additional sections.

For example, if he gives details about his school/college, he will be more likely to get friend's recommendations based on his locality and graduated from the same school/college.

\subsection{SEARCH PEOPLE}

The interface for "Search People" module has been designed keeping in mind of user's ease to access i.e. to grow his/her professional connections which is based on user's location by keeping his location mode "On". A user can look for other users and can get introduced. We have included three submodules under Search People interface i.e. Search People by Events, Skills and Communities which will really help the users to filter the search results in easy way with minimal input from their side.

It is important to note that the proposed model depends on the user's location. Based on the user's location, the search results will be generated and only those profile of users will be in search results who are of the same location.

1. Search People By Events: The search results for this sub-module is based on the people who has attended that event provided the event is held at the same venue, time and date. The fig. 6 shows the way to search people by events. Here, the user will input the details of time, date and venue in the userfriendly with minimal input design where the user only has to select and provide the details. 
All the profiles of the users, who attended the event will be shown as Search Results as shown in fig. 9. After getting the search results, one can send an introduction message to other user who attended the event. It is quite obvious that the people who attended the event may have common interests and with this social networking platform one can easily get in touch with the people who attended the event.

2. Search People By Skills: In these days, the searching for people based on skills has become quite popular especially for professional social networking platforms. The fig. 7 shows the way to search people by skills where a drop-down list will come and the list of skills will be displayed.

The user can easily select the skill of which he is looking for and will get the instant results of the profiles of users as shown in fig. 9. With the search results, one can send the introduction message to other user of the required skills.

3. Search People By Communities: In a given location, there happens to be many clubs as well as associations and groups. People of the same community share common interests and goals. With this social networking platform, it will easily help a user to stay in touch with the other users belonging to the same community. The fig. 8 shows the way to search people by communities. The user can select the community from the drop down list and the search results will list the user profiles belonging the community. With the search results, one can send the introduction message to other users of the same community.

\subsection{PRIVACY}

There are many issues with the current social networking platforms regarding privacy, data thefts, viruses, information misuse etc. The interface as shown in fig. 10, provides an easy interface where a user can select on/off option. From the figure it is quite clear that, the user can turn on/off his location, skills as well as communities and events information.

Turn on will display all details in public mode whereas Turn off will display all details to the user and to whom he is connected.

When the user will turn off his location, skills, complete profile, the user's profile will not be shown in any search results. Only to the users to whom he is connected, can view his profile.

Even when a User 1 is sending an introduction message to User 2, the User 2 has the choice to accept/deny the friend request from User 1.

\section{FUNCTIONALITY AND FEATURES}

The core functionality and features of the proposed model is as follows:

1. Instant Search Results: Suppose there was a technical conference which was held two days ago .It is quite obvious that people attending the conference must have common interests or goals. But, in order to get introduced with the people is quite tough at the time of conference due to crowd and other factors. Our model will help to cope up with this problem. After the conference, i.e. after two days, one can search for people by events by entering the details such as date, time and venue as shown in fig 6.

All the profiles of the users, who attended the conference, will be shown as Search Results as shown in fig 9. After getting the search results, one can send an introduction message to other user who attended the conference.

2. Portability: The portability is a very important component while designing an information system. The proposed model is designed keeping in mind of mobile users where they can easily make out the use of the social networking platform in a go even they are outside of their office/home.

3. User Friendly Interface: The interface of the proposed model is very easy to use. Here, the user doesn't have to type email-id, user's name or other info during the searching of other users. He can easily select the search parameters i.e. by events, skills, communities and can look for users. Also, the privacy interface has been provided with On/Off option.

A user can easily On/Off his details without any complications.

\section{EXPERIMENTAL RESULTS}

Each figure (figure 1, 2, 3, 4, 5, 6, 7, 8, 9, 10) that is shown below represents the experimental results of the proposed model.

Consider the scenario, when a company HR is looking for Android developers in his area. In the interface provided for search people by skills as shown in fig 7., he can select the "Android" skill from the list of skills. After that, in the search results as shown in fig 9., he will get the list of user's profiles with the Android Development skills that he was looking for and later he can send an introductory message for a meet-up or an interview. 


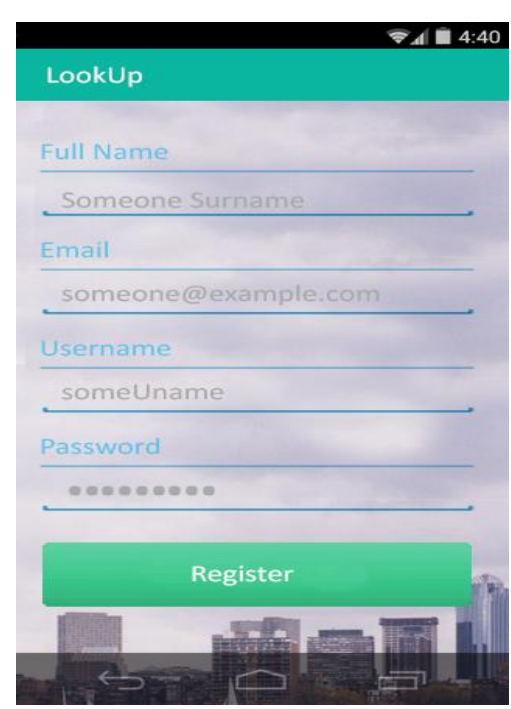

Fig 1. User Sign Up

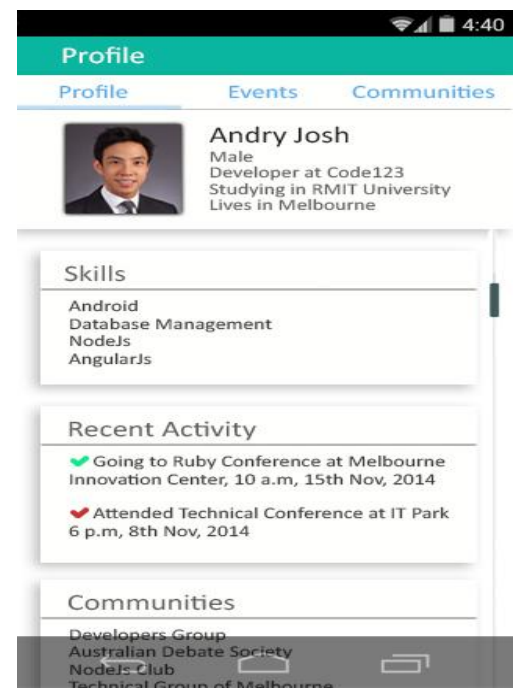

Fig 2. User Profile

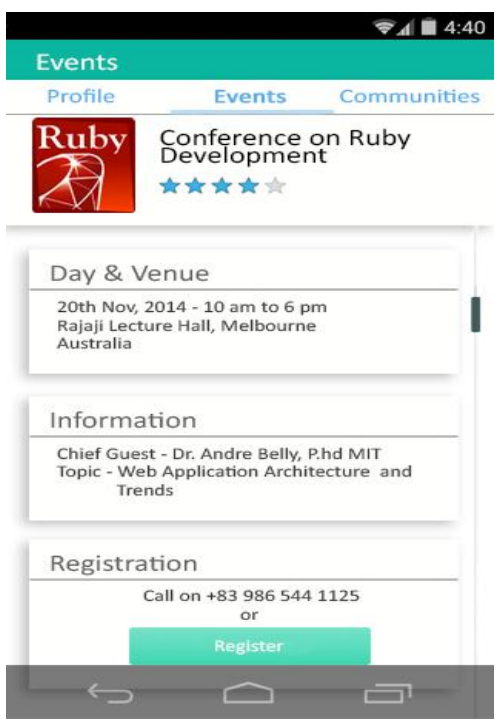

Fig 3. Event Information

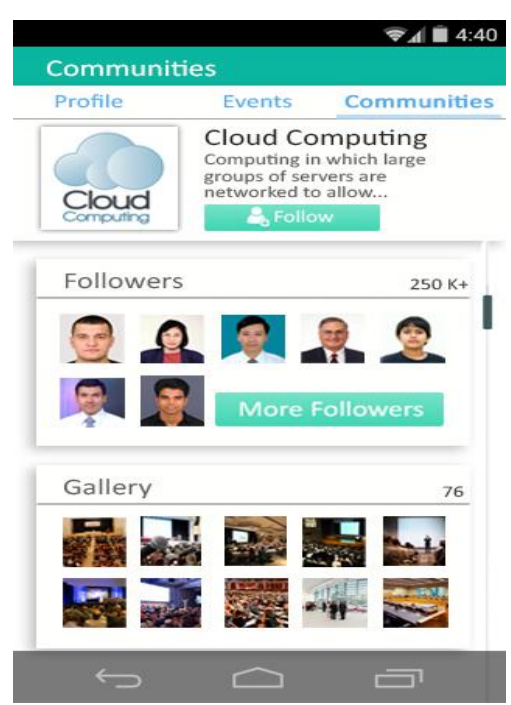

Fig 4. Communities Information

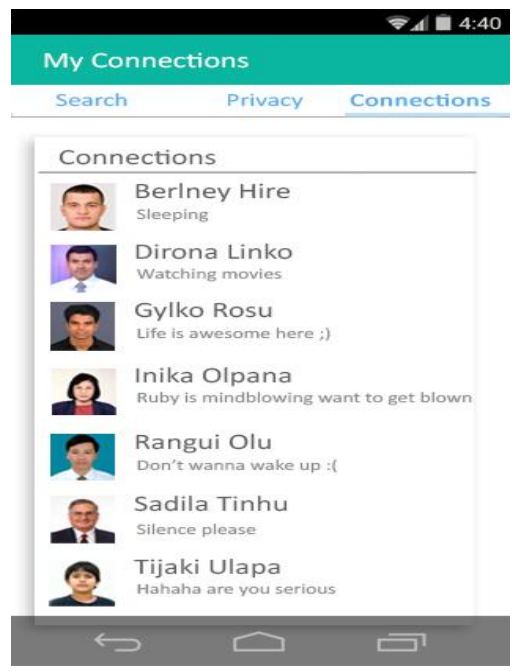

Fig 5. User Connections

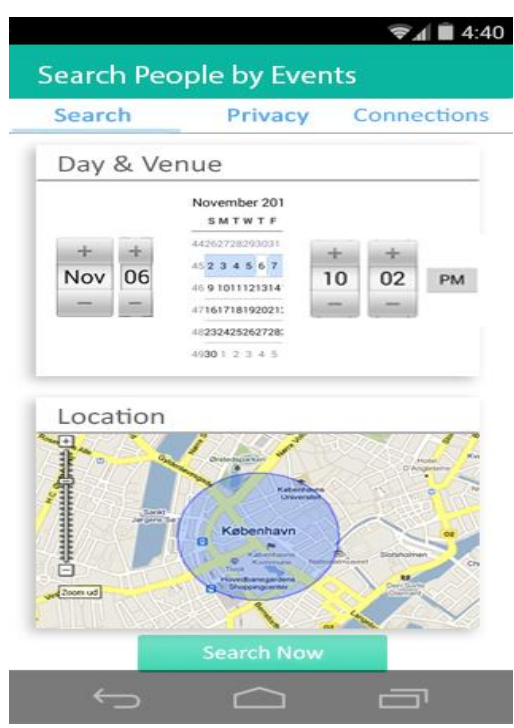

Fig 6. Filtering by Date, Time and Location 


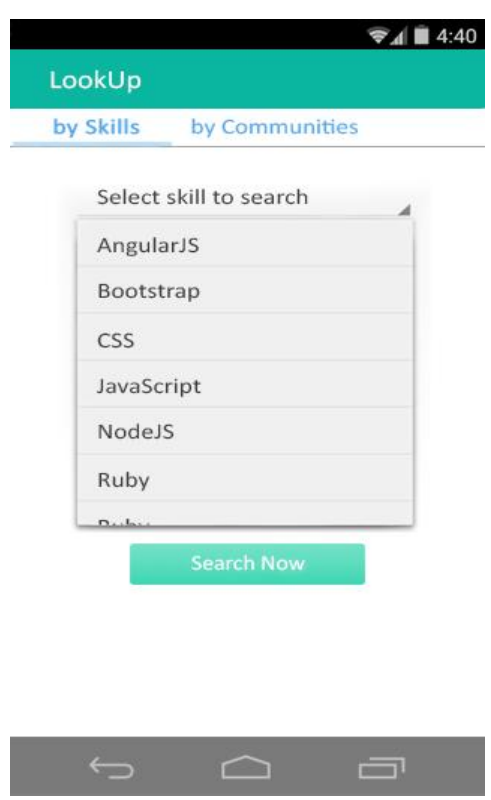

Fig 7. Filtering by Skills

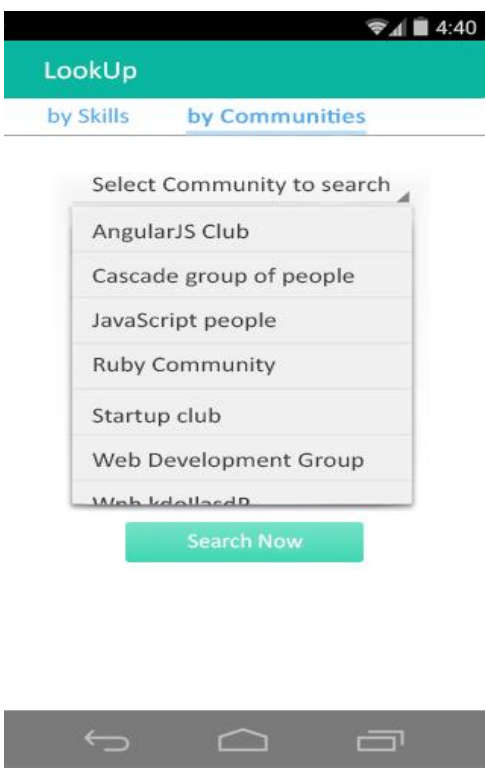

Fig 8. Filtering by Communities

\section{CONCLUSIONS}

The above proposed model mainly focuses on the interface design as well as the functional design of our new Social Networking platform. It covers all the major aspects that is required in the searching of people of desired skills as well as interests, getting introduced to people and also keeping in mind of providing privacy user-friendly feature.

The proposed model mainly focuses on reducing the amount of time spent by the user and providing instant results with user satisfaction. The interface is thus designed keeping in mind of meeting user's business goals thus staying focused on work, reducing time spent online, thus promoting useful usage of the proposed model.

The reliability of a social networking information system is judged by the following factors:

1. Instant Results.

2. Accuracy.

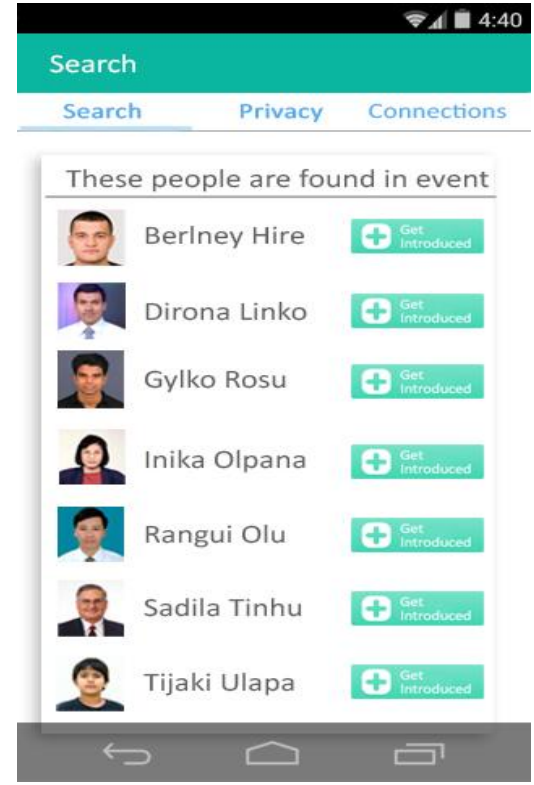

Fig 9. Search Results

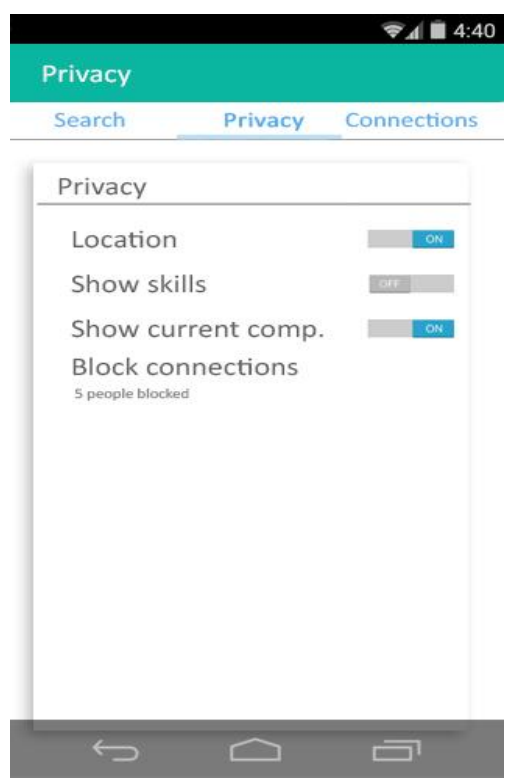

Fig 10. Privacy Interface

3. Consistency.

4. Ease-to-Use.

5. Portability.

6. Privacy.

This model mainly provides theoretical reference for the realization of our new Social Networking platform.

\section{REFERENCES}

[1] Rios-Aguilar S, Llorens-Montes F-J, Pedromingo-Suárez, A, "A mobile business information system for the control of local and remote workforce through reactive and behavior-based monitoring", Expert Systems with Applications, Volume 42, Issue 7, 1 May 2015, Pages 3462-3469, Elsevier.

[2] Huiping Cao, Shan Wang, Lingwei Li, "Location dependent query in a mobile environment" ,Information Sciences, Volume 154, Issues 1-2, August 2003, Pages 
71-83,Elsevier.

[3] Hopfner, H., Nakata, K., "On Teaching Mobile Databases and Information Systems", Databases, 2007. BNCOD '07. 24th British National Conference, Pages 4858, IEEE Explorer Digital Library.

[4] Jonathan Simon, "Head First Android Development," in O'Reilly Media, Inc; First Edition, October (2011).

[5] Pant, S., Hsu, C., "Strategic Information Systems Planning: A Review, Information Resources Management", Association International Conference, May 21-24, Atlanta (1995).

[6] Ben Shneideran, Maxin Cohen, Steve M. Jacobs, "Designing the User Interface - Strategies for Effective Human Computer Interaction $5^{\text {th }}$ Edition", 2010.
[7] Furnell R, Scott P, "Can Social Networking improve Project Management? An exploratory study of UK professional experience", 2014 International Conference on Information Society, London, United Kingdom, Article Number 7009031 Pages 152-156.

[8] Azizi Yahaya, Gooh Mo Lee, Haliman Ma'alip, Dayang Ku Zainab Pg Hj Tuah, Zeliha Mohammad Ali, Kamariah Hj Kasah, Zarifa Binti Mohd Sis, "The Impact of Social Networking on Behavioural Development among Secondary School Students", Computational Intelligence in Information Systems, Advances in Intelligent Systems and Computing, Volume 331, 2015, Pages 191-199.

[9] Klein A, Ahlf H, Sharma V, "Social activity and structural centrality in online social networks", Telematics and Informatics, Volume 32, Issue 2, 1 May 2015, Pages 321-332. 